\title{
Magnetic anisotropy in the excited states of low symmetry lanthanide complexes $\dagger$
}

\author{
Liviu Ungur* and Liviu F. Chibotaru* \\ Received 22nd August 2011, Accepted 13th October 2011 \\ DOI: $10.1039 / \mathrm{c} 1 \mathrm{cp22689d}$
}

\begin{abstract}
$A b$ initio investigation of multiplet spectrum of lanthanides in archetypal coordination geometries shows an unexpected regular structure consisting of (i) mirror symmetry of anisotropic magnetic properties of doublet states, (ii) high magnetic axiality of low-lying and high-lying doublets, comparable to complexes with ideal axial symmetry, and (iii) the strong rotation of the anisotropy axes of individual doublets. The obtained high axiality of the ground doublet states explains the SMM behaviour of low-symmetry lanthanide complexes.
\end{abstract}

Understanding the factors determining the formation of efficient barriers of blocking of magnetization in single-molecule magnets (SMMs) is of primary importance for the fast advance of this new research area ${ }^{1-4}$ towards various applications in storage and information processing technologies. ${ }^{5-9}$ A key condition for achieving the slow relaxation of magnetization in such complexes is the effective involvement of the anisotropy of the metal sites. In this respect the lanthanide-containing complexes look especially attractive due to their large spin-orbit coupling compared to the crystal-field splitting of the magnetic $4 \mathrm{f}$-shell, ${ }^{10}$ which is the reason for their current intensive studies. ${ }^{11}$ One of the conclusions reached in these studies is that the excited states of the lanthanide ions play a crucial role in the relaxation process of both mononuclear ${ }^{12-16}$ and polynuclear compounds, which can be either mixed $4 \mathrm{f}-3 \mathrm{~d}^{17-29}$ or of pure lanthanide type. ${ }^{30-37}$ Here the anisotropic properties of the excited Kramers and Ising doublets of lanthanide ions in several representative complexes are studied ab initio ${ }^{38,39}$ within the CASSCF/RASSI/SINGLE_ANISO approach as implemented in MOLCAS 7.6 version. ${ }^{40}$ The results show clear regularities in the variations of anisotropy axes with the energy of excited doublets in low-symmetric Ln fragments, which could be in principle probed by optically-detected EPR. ${ }^{41-43}$

The simplest case of an ideal axial symmetry is achieved in a diatomic complex such as, e.g., $[\mathrm{DyO}]^{+}$. Due to a perfect axial symmetry, all electronic states are characterized by a definite

Division of Quantum and Physical Chemistry and INPAC-Institute of Nanoscale Physics and Chemistry, Katholieke Universiteit Leuven,

Celestijnenlaan 200F, 3001, Leuven, Belgium.

E-mail:Liviu.Ungur@chem.kuleuven.be,

Liviu.Chibotaru@chem.kuleuven.be; Fax: +32 1632 7992;

Tel: +3216327424

$\dagger$ Electronic supplementary information (ESI) available. See DOI: $10.1039 / \mathrm{c} 1 \mathrm{cp} 22689 \mathrm{~d}$
Table 1 Energies $\left(\mathrm{cm}^{-1}\right)$ and main values of the $g$ tensors of $[\mathrm{DyO}]^{+}$ in the lowest Kramers doublets

\begin{tabular}{llrll}
\hline KD & $\left|M_{J}\right|$ & $E / \mathrm{cm}^{-1}$ & \multicolumn{1}{l}{$g_{\|}$} & $g\left(\mathrm{Dy}^{3+}\right)^{a}$ \\
\hline 1 & $15 / 2$ & 0.0 & 19.908 & 20.000 \\
2 & $13 / 2$ & 767.2 & 16.869 & 17.333 \\
3 & $11 / 2$ & 1371.5 & 14.100 & 14.667 \\
4 & $9 / 2$ & 1783.5 & 11.541 & 12.000 \\
5 & $7 / 2$ & 2013.0 & 9.064 & 9.333 \\
6 & $5 / 2$ & 2108.8 & 6.562 & 6.667 \\
7 & $1 / 2$ & 2132.1 & 1.337 & 1.333 \\
8 & $3 / 2$ & 2132.5 & 3.984 & 4.000 \\
\multicolumn{2}{l}{ From ref. 44.} & & & \\
\hline
\end{tabular}

projection $M_{J}$ of the total angular momentum on the molecular axis. Since $[\mathrm{DyO}]^{+}$has an odd number of electrons, all these states are Kramers doublets, ${ }^{44}$ with two components corresponding to $\pm\left|M_{J}\right|$. Table 1 shows the ab initio calculated energies and $g_{\|}$for the lowest eight Kramers doublets (characterized by an effective spin $S=1 / 2$ ), originating from the ground atomic multiplet ${ }^{6} H_{15 / 2}$ of $\mathrm{Dy}^{3+}$, at the equilibrium geometry of the complex (see the ESI $\dagger$ ). Note that in this complex $g_{\perp}=0$ for all Kramers doublets except $M_{J}= \pm 1 / 2$. Thus the Kramers doublet for $\left|M_{J}\right|=1 / 2$ has $g_{\perp}=10.54$ in $[\mathrm{DyO}]^{+}\left(10.67\right.$ in $\left.\mathrm{Dy}^{3+}\right)$. Due to a perfect axiality of the Kramers doublets up to the highest one, the transversal magnetic field can induce a tunneling splitting in $[\mathrm{DyO}]^{+}$only in the high order of perturbation theory, following the path shown by arrows in Fig. 1.

On the other hand, due to the high energy of the barrier $\left(>3000 \mathrm{~K}\right.$ ) its population at $300 \mathrm{~K}$ is only $3.5 \times 10^{-5}$, which means that the thermal relaxation of magnetization will be efficiently blocked also at room temperature. We note that the obtained very high barrier is the result of a remarkably short bond length (1.74 ̊). Contrary to conventional lanthanide complexes with eight or nine atoms surrounding the $\mathrm{Ln}^{3+}$ ion, in $[\mathrm{LnO}]^{+}$the high-order chemical bond is achieved (see the ESI $\dagger$ ).

Contrary to this example, the multiplets in axially symmetric complexes with non-linear geometry are not characterized by a definite $M$ anymoret but their properties are entirely defined by the corresponding irreducible representation (irrep) ${ }^{45}$ of the symmetry group of the complex. Applying symmetry-based selection rules (which predict when a matrix element of an operator is zero on symmetry grounds ${ }^{45}$ ) to the components of the magnetic moment, we can specify all cases when a complex is characterized by a perfect axiality. Thus we find that the 


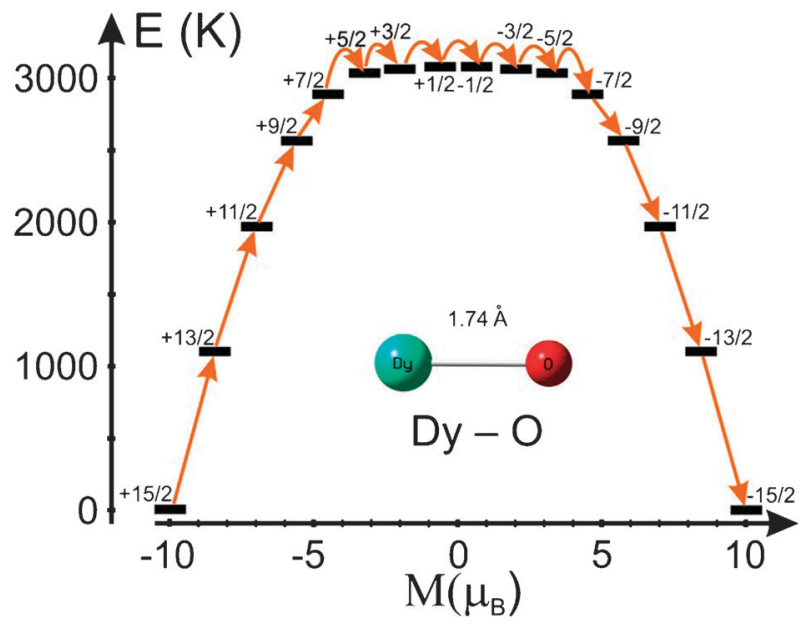

Fig. 1 Barrier for reversal of magnetization in $[\mathrm{DyO}]^{+}$at equilibrium geometry. Horizontal lines are energy levels (Table 1) and the arrows show the path for the reversal of magnetization.

Kramers doublet is perfectly axial $\left(g_{\perp}=0\right)$ only if the irrep $E_{\perp}$ after which transform the two transversal components of the magnetic moment (denoted by $E$ or $E_{1}$ in different groups ${ }^{46}$ ) is not contained in the symmetrized square of the irrep $E_{\mathrm{KD}}$ after which transforms this Kramers doublet, $E_{\perp} \notin\left[E_{\mathrm{KD}}^{2}\right]$. These cases are listed in Table 2. As an example, consider the ground Kramers doublet in a double-decker complex $\left[\mathrm{Dy}(\mathrm{Pc})_{2}\right]^{-13}$ in an ideal symmetry $D_{4 \mathrm{~d}}$. The corresponding wave functions are $M_{J}=$ $\pm 13 / 2$ in the crystal-field approximation, ${ }^{13}$ which transform under the symmetry operations as $M=\mp 3 / 2$, respectively, i.e. correspond to the irrep $E_{3 / 2}$. The latter corresponds to a perfectly axial doublet according to Table 2. An opposite example of a Kramers doublet without perfect axiality is given by the irrep $E_{1 / 2}$ in all relevant groups (e.g. the KD nr. 7 in Table 1).

Similarly, the Ising doublet (in the case of non-Kramers Ln ions) is perfectly axial (i.e., degenerate, $\Delta_{\mathrm{CF}}=0$ ) only if its two components transform after a twofold degenerate irrep $\left(E, E_{1}\right.$, $E_{2}$, in different groups ${ }^{46}$ ). The possible situations are listed in Table 2. As an example consider the ground doublet in the double decker complex $\left[\mathrm{Tb}(\mathrm{Pc})_{2}\right]^{-13}$ in an ideal symmetry $D_{4 \mathrm{~d}}$.
The corresponding wave functions are $M_{J}= \pm 6$ in the crystal field approximation, ${ }^{13}$ which transform under symmetry operations as $M=\mp 2$, respectively, i.e. correspond to the degenerate irrep $E_{2}$ of the $D_{4 \mathrm{~d}}$ group.

As examples of ground state Ising doublets without perfect axiality $\left(\Delta_{\mathrm{CF}} \neq 0\right)$ can be mentioned the complexes $\mathrm{Mn}_{12} \mathrm{acac}^{1}$ and $\mathrm{Dy}_{4} \mathrm{Cr}_{4},{ }^{27}$ whose two wave functions transform after non-degenerate irreps $A_{1}$ and $A_{2}$, respectively, even in an ideal $D_{2 \mathrm{~d}}$ symmetry. Nevertheless, in all cases discussed above the anisotropy axis coincides with the main symmetry axis of the complex. The real structure of the complexes is always slightly distorted from the high symmetry geometry (this is the case, e.g., in highly symmetric double-decker phthalocyanine and polyoxometalate lanthanide complexes ${ }^{12-16}$ ) and, therefore, $g_{\perp}, \Delta_{\mathrm{CF}}$ become slightly non-zero even for doublets otherwise expected to be perfectly axial. The extent of the departure from a perfect axiality is very sensitive to the environment and is manifested in opening/closing of relaxation channels by varying the later. An example of this kind is the neutral double-decker phthalocyanine complex of terbium showing strongly varying activation energy for magnetic relaxation in different environments. ${ }^{13,16}$

In an opposite limit of single-Ln complexes and fragments with geometry far from any high symmetry point, the anisotropy of excited doublets displays an unexpected regular behavior. The low site-symmetry is typical for polynuclear complexes ${ }^{17-37}$ from which we further consider two representative examples. In a molecular $\mathrm{Dy}_{3}$ triangle, ${ }^{30,32,33}$ two dysprosium ions are surrounded by eight oxygens while one of them (Dy3 site) is surrounded either by seven oxygens and one chlorine or six oxygens and two chlorines. ${ }^{30}$

The polyhedron formed by these eight atoms corresponds to a distorted trigonal dodecahedron or a distorted square antiprism. Because the surrounding oxygens are of quite different nature $\left(\mu_{3}-\mathrm{O}, \mu_{3}-\mathrm{OH}, \mu_{2}-\mathrm{OH}_{2}\right)$, the crystal field felt by the dysprosium ion is actually of a much lower symmetry than one could infer from the structure of the first coordination sphere. In the previous ab initio studies ${ }^{32,39}$ it was found that the anisotropy axes in the ground Kramers doublet of the dysprosium sites lie almost in the $\mathrm{Dy}_{3}$ plane.

Table 2 Irreps of point groups for which a perfect axiality of a Kramers doublet $\left(g_{\perp}=0\right)$ and of an Ising doublet $\left(\Delta_{\mathrm{CF}}=0\right)$ is achieved

\begin{tabular}{|c|c|c|c|}
\hline Point group & Main symm. axis & $\begin{array}{l}\text { Even number of electrons } \\
\text { Ising doublet }\end{array}$ & $\begin{array}{l}\text { Odd number of electrons } \\
\text { Kramers doublet }\end{array}$ \\
\hline $\begin{array}{l}C_{2}, C_{2 \mathrm{v}}, C_{2 \mathrm{~h}} \\
C_{3} \\
C_{4} \\
S_{4}, D_{2 \mathrm{~d}} \\
D_{2}, D_{2 \mathrm{~h}} \\
D_{3 \mathrm{~h}}, C_{3 \mathrm{~h}} \\
D_{3}, C_{3 \mathrm{v}} \\
D_{4}, C_{4 \mathrm{v}}, D_{2 \mathrm{~d}} \\
C_{4 \mathrm{~h}}, D_{4 \mathrm{~h}} \\
D_{5}, C_{5 \mathrm{v}}, C_{5} \\
C_{5 \mathrm{~h}}, D_{5 \mathrm{~h}} \\
D_{6}, C_{6 \mathrm{v}}, C_{6} \\
C_{6 \mathrm{~h}}, D_{6 \mathrm{~h}} \\
S_{6}, D_{3 \mathrm{~d}} \\
D_{8}, C_{8 \mathrm{v}}, D_{4 \mathrm{~d}}, D_{8 \mathrm{~h}} \\
D_{\infty}, C_{\infty \mathrm{v}}, D_{\infty \mathrm{h}} \\
D_{\infty \mathrm{v}}\end{array}$ & $\begin{array}{l}C_{2} \\
C_{3} \\
C_{4} \\
S_{4} \\
C_{2} \\
C_{3} \\
C_{3} \\
C_{4} \\
C_{4} \\
C_{5} \\
C_{5} \\
C_{6} \\
C_{6} \\
S_{6} \\
C_{8} \\
C_{\infty} \\
C_{\infty}\end{array}$ & $\begin{array}{l}\bar{E} \\
E \\
E \\
\overline{E^{\prime}}, E^{\prime \prime} \\
E \\
E \\
E_{\mathrm{g}}, E_{\mathrm{u}} \\
E_{1}, E_{2} \\
E_{1}^{\prime}, E_{2}^{\prime}, E_{1}^{\prime \prime}, E_{2}^{\prime \prime}, \\
E_{1}, E_{2} \\
E_{1 \mathrm{~g}}, E_{1 \mathrm{u}}, E_{2 \mathrm{~g}}, E_{2 \mathrm{u}} \\
E_{\mathrm{g}}, E_{\mathrm{u}} \\
E_{1}, E_{2}, E_{3} \\
\Pi, \Delta, \Phi \\
\Pi_{\mathrm{g}}, \Pi_{\mathrm{u}}, \Delta_{\mathrm{g}}, \Delta_{\mathrm{u}}, \Phi_{\mathrm{g}}, \Phi_{\mathrm{u}}, \ldots\end{array}$ & $\begin{array}{l}- \\
- \\
- \\
\overline{-} \\
E_{3 / 2} \\
E_{3 / 2} \\
- \\
E_{3 / 2}, E_{5 / 2} \\
E_{3 / 2}, E_{5 / 2} \\
E_{3 / 2} \\
E_{3 / 2} \\
E_{3 / 2} \\
E_{3 / 2}, E_{5 / 2}, E_{7 / 2}, \ldots \\
E_{3 / 2}, E_{5 / 2}, \ldots \\
E_{3 / 2}, E_{5 / 2}, E_{7 / 2}, \ldots\end{array}$ \\
\hline
\end{tabular}


Table 3 Energies $\left(\mathrm{cm}^{-1}\right)$ and main values of the $g$ tensors of the lowest Kramers doublets at the Dy3 site of the dysprosium triangle

\begin{tabular}{lrlll}
\hline KD & Energy & $g_{X}$ & $g_{Y}$ & $g_{Z}$ \\
\hline 1 & 0.000 & 0.008 & 0.034 & 19.667 \\
2 & 103.476 & 0.398 & 0.645 & 17.750 \\
3 & 141.038 & 3.495 & 3.670 & 11.366 \\
4 & 171.100 & 2.214 & 4.969 & 11.748 \\
5 & 208.691 & 0.069 & 3.223 & 14.098 \\
6 & 228.586 & 1.215 & 3.047 & 16.450 \\
7 & 287.051 & 0.104 & 0.281 & 18.743 \\
8 & 395.074 & 0.002 & 0.008 & 19.556 \\
\hline
\end{tabular}

Now these studies are extended over the excited Kramers doublets originating from the ground atomic multiplet ${ }^{6} \mathrm{H}_{15 / 2}$ of $\mathrm{Dy}^{3+}$, by performing a new series of $a b$ initio calculations (see the ESI $\dagger$ ). Table 3 shows the results of calculations for the main values of $g$ tensors of the eight Kramers doublets on the Dy3 site (Fig. 2); the entire $g$ tensors in the molecular coordinate frame and the angles between main magnetic axes of different doublets are given in Tables S2-S8 (ESI $\dagger$ ). We can see from Table 3 that the $g$ tensor for the ground Kramers doublet (1) is very axial, with the transversal components $g_{X}$, $g_{Y}<0.01$. At the same time $g_{Z}$, corresponding to the main anisotropy axis, is close in value to $g_{\|}$for the ground Kramers doublet of $[\mathrm{DyO}]^{+}$(Table 1). This means that the ground Kramers doublet of Dy3 site is close to the axial Kramers doublet with $M_{J}= \pm 15 / 2$ (Table 1) when its quantization axis is taken along the main anisotropy axis of Dy3 (Fig. 2). The next Kramers doublet on the Dy3 site (2) is also strongly anisotropic, with $g_{Z}$ close to the value corresponding to the second Kramers doublet on $[\mathrm{DyO}]^{+}$(see Table 1), i.e. to $M_{J}=$ $\pm 13 / 2$. However now the main anisotropy axis is rotated with respect to Kramers doublet 1 by $c a .42^{\circ}$ (Fig. 2). The axiality of the $g$ tensors gradually decreases up to the Kramers doublets 3 and 4 (Table 3), then it starts to increase again reaching for the highest doublet (8) the value comparable for the doublet 1 , i.e. being again close to $M_{J}= \pm 15 / 2$. Such a

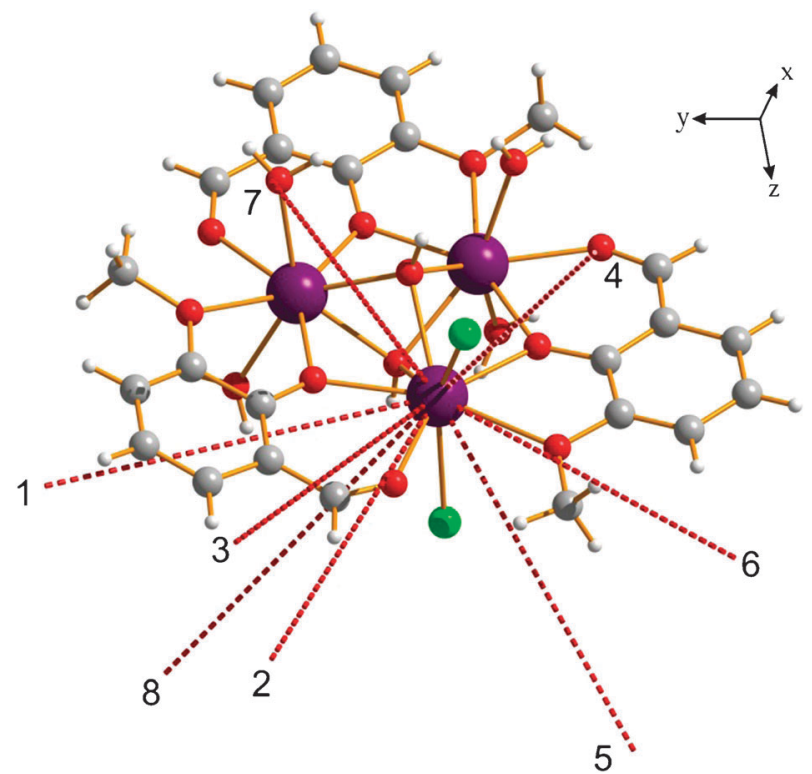

Fig. 2 Anisotropy axes in the lowest Kramers doublets at the Dy3 site of the dysprosium triangle. mirror symmetry in the magnetic properties of the lowest Kramers doublets is found in sharp contrast with the perfectly axial systems (Table 1). Moreover, the directions of the main anisotropy axes rotate when going to higher doublets (Fig. 2). The described situation with the magnetic anisotropy in excited Kramers doublets is completely similar for the other two sites, Dy1 and Dy2 (Tables S3-S6, ESI $\dagger$ ), despite their different electronic structures (Table S2, ESI $\dagger$ ).

These findings have direct implications for the barriers of reversal of magnetization at low symmetry Dy sites. Fig. 3 shows the matrix elements of magnetic moment operators (in the coordinate system related to the anisotropy axes in the ground state) between the components of the two lowest Kramers doublets of Dy3 site. We can see that the matrix elements of $\mu$ are non-negligible between all four components. In particular, they can connect both components of the lowest Kramers doublet to the same component of the excited Kramers doublet, which makes operative the Orbach and Raman relaxation processes $\S$ via this doublet. ${ }^{44}$ This is partly due to non-coinciding anisotropy axes in the ground and the first excited Kramers doublets (Fig. 2). Indeed, even if the two Kramers doublets would ideally correspond to $M_{J}= \pm 15 / 2$ and $M_{J}= \pm 13 / 2$, respectively, the matrix element of the magnetic moment $\mu$ between the components $+15 / 2$ and $-13 / 2$ will be non-zero due to the non-coincidence of the anisotropy axes (the same for $-15 / 2$ and $+13 / 2$ ). This is not the case in complexes with perfect axial symmetry (Fig. 1), where the anisotropy axis is the same in all Kramers doublets. Furthermore, given that the angle between the anisotropy axes in doublets 1 and 2 is $42^{\circ}$, these matrix elements are nonnegligible and larger than the matrix elements connecting the two components of the same Kramers doublet (Fig. 3). We may conclude that the non-coincidence of the anisotropy axes (i) sets the height of the barrier of reversal of magnetization to the energy of the first excited Kramers doublet and (ii) enhance the Orbach and Raman relaxation processes via this doublet.

As an example of non-Kramers ions we consider terbium fragments from recently synthesized $\mathrm{Tb}_{3}$ complexes (Fig. 4). ${ }^{37}$ As Fig. 4 shows, the coordination of each terbium ion includes six oxygens and two nitrogens which moreover are not equivalent from the point of view of chemical bonding.

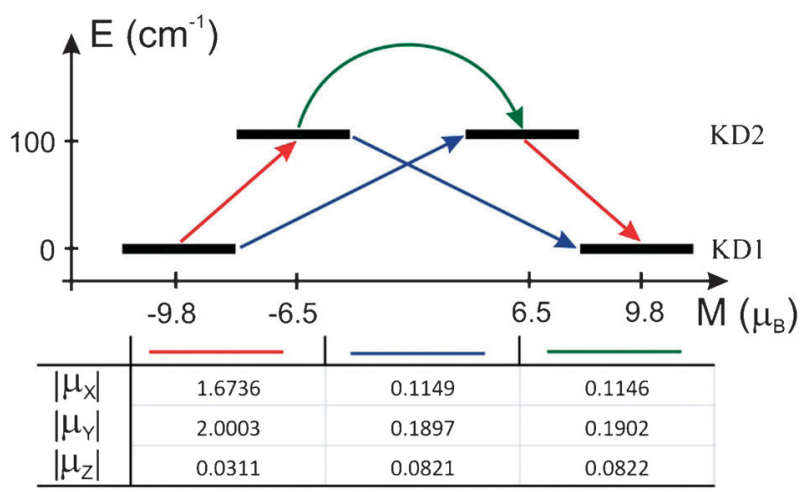

Fig. 3 Paths of reorientation of magnetization (arrows) on individual Dy sites through the first excited Kramers doublet. The table gives absolute values of the matrix elements of the magnetic moments $\mu_{X}$, $\mu_{Y}, \mu_{Z}$ between the lowest Kramers doublets at the Dy3 site. 


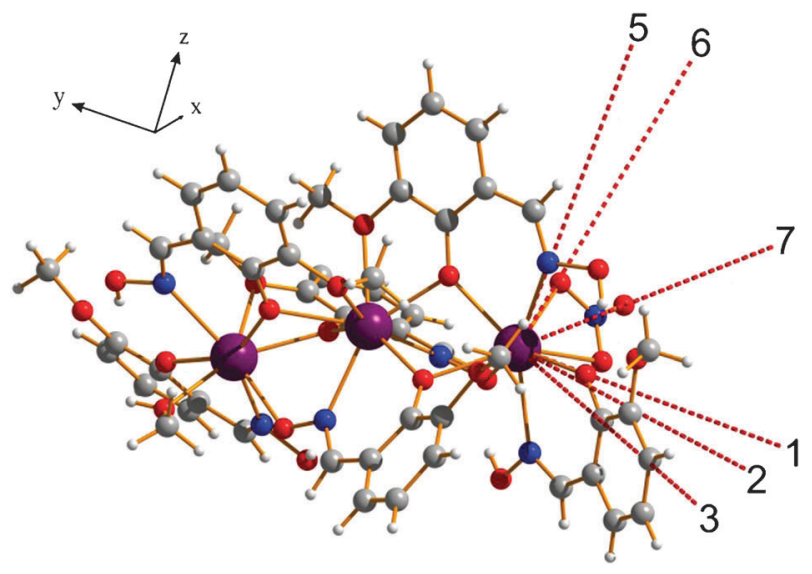

Fig. 4 Anisotropy axes in the lowest doublets at the Tb3 site of the $\mathrm{Tb}_{3}$ complex. $^{37}$

Table 4 Energies $\left(\mathrm{cm}^{-1}\right)$ and the $g_{Z}$ values ${ }^{a}$ of the lowest Ising doublets (ID) at the $\mathrm{Tb} 3$ site of the $\mathrm{Tb}_{3}$ complex

\begin{tabular}{lrr}
\hline ID & Energy & $g_{Z}$ \\
\hline 1 & 0.000 & 17.837 \\
& 0.090 & 14.711 \\
2 & 106.225 & \\
& 107.496 & 11.853 \\
3 & 171.446 & \\
& 178.386 & \\
4 & 214.780 & 9.904 \\
5 & 235.191 & 14.113 \\
& 242.988 & 17.685 \\
6 & 275.819 & \\
7 & 277.512 & \\
& 485.772 & \\
& 485.818 & \\
${ }^{a} g_{X}=g_{Y}=0$ according to Griffith's theorem & \\
& &
\end{tabular}

Therefore, as in the case of Dy sites in the dysprosium triangle, we expect each terbium ion to experience a crystal field far from any symmetry. The electronic structure of individual $\mathrm{Tb}$ fragments has been already investigated ab initio, ${ }^{37}$ here we concentrate on the anisotropic properties of the excited states. Table 4 shows the calculated energies of multiplets for the $\mathrm{Tb} 3$ center originating from the ground atomic multiplet ${ }^{7} \mathrm{~F}_{6}$ of $\mathrm{Tb}^{3+}$. We can see that the 13 energy levels can be grouped in six doublets and one nondegenerate level, in full correspondence with the multiplet structure arising in the case of perfect axial symmetry (six degenerate Ising doublets with $M_{J}= \pm n, n=1,6$ and one nondegenerate with $M_{J}=0$ ). The last column of Table 4 gives the values of $g_{\|}$for the doublets $\left(g_{\perp}=0\right.$ according to Griffith's theorem $\left.{ }^{44}\right)$. As in the case of dysprosium fragment considered above, we can see a mirror symmetry for the lower and higher doublets with respect to both their tunneling gaps and the $g_{Z}$ factor. The lowest and highest doublets are again the most axial ones, corresponding in the present case to the smallest tunneling splitting among the doublets (Table 4 ) and $g_{\|}$close to the value for the axial doublet $M_{J}= \pm 6\left(g_{\|}=18\right)$. The second highest and lowest doublets roughly correspond to $M_{J}= \pm 5\left(g_{\|}=15\right)$. A similar situation takes place for another two terbium centers in this complex, Tb1 and Tb2 (Tables S10-S14, ESI $\dagger$ ).
To conclude, we have investigated $a b$ initio the magnetic anisotropy in the excited multiplets of several representative lanthanides. The mononuclear fragments with archetypal coordination, whose crystal field is expected to be far from any symmetry, show surprising regularities of magnetic anisotropy in the excited multiplets. The similar behavior of magnetic anisotropy at Dy and $\mathrm{Tb}$ centers from complexes investigated here points to some common basic features of the crystal field in these fragments which are expected to persist also in other lanthanide complexes and fragments without symmetry. Finding the main components of the crystal field responsible for this behavior and the reason for their persistence in structurally different Ln fragments is an important task for future ab initio investigations. Finally, we stress that the almost perfect axiality of the ground doublet state of investigated low-symmetry lanthanide centers is an important feature enabling them functioning as single-molecule magnets, especially, in polynuclear complexes where they do not have as a rule any site symmetry.

\section{Note added after first publication}

This article replaces the version published on 25th October 2011, which contained errors in the first line of the final paragraph of the first page.

\section{Notes and references}

$\ddagger$ In the crystal field approximation, treating the 4f shell only, the multiplets will still be characterized by definite $M$ if the order of the main symmetry axis is higher than six (e.g., in complexes with ideal $D_{4 \mathrm{~d}}$ symmetry, where the main magnetic axis is $S_{8}$ ). In such cases all multiplets with $M>1 / 2$ are always perfectly axial, i.e. $g_{\perp}=0$ for Kramers doublets, while the crystal field splitting is $\Delta_{\mathrm{CF}}=0$ for Ising doublets (in complexes with even number of electrons). However, $M$ ceases to be good quantum number when the metal-ligand covalency is taken into account, as is the case in quantum chemistry calculations. $\S$ Although Raman and Orbach processes are not determined directly by the matrix elements of the magnetic moments, the non-negligible values of the latter serve as an indicator of the relevance of these relaxation processes.

1 R. Sessoli, D. Gatteschi, A. Caneschi and M. A. Novak, Nature, 1993, 365, 141-143.

2 G. Christou, D. Gatteschi, D. N. Hendrickson and R. Sessoli, MRS Bull., 2011, 25, 66-71.

3 D. Gatteschi and R. Sessoli, Angew. Chem., Int. Ed., 2003, 42, 268-297.

4 D. Gatteschi, R. Sessoli and J. Villain, Molecular Nanomagnets, Oxford University Press, Oxford, 2006.

5 M. Affronte, F. Troiani, A. Ghirri, A. Candini, M. Evangelisti, V. Corradini, S. Carretta, P. Santini, G. Amoretti, F. Tuna, G. Timco and R. E. P. Winpenny, J. Phys. D: Appl. Phys., 2007, 40, 2999-3004.

6 L. Bogani and W. Wernsdorfer, Nat. Mater., 2008, 7, 179-186.

7 E. M. Chudnovsky, Science, 1996, 274, 938-939.

8 D. D. Awschalom and D. P. Divincenzo, Phys. Today, 1995, 48, 43-48.

9 M. N. Leuenberger and D. Loss, Nature, 2001, 410, 789-793.

10 C. Benelli and D. Gatteschi, Chem. Rev. (Washington, DC, U. S.), 2002, 102, 2369-2387.

11 R. Sessoli and A. K. Powell, Coord. Chem. Rev., 2009, 253, $2328-2341$.

12 N. Ishikawa, M. Sugita, T. Ishikawa, S. Koshihara and Y. Kaizu, J. Am. Chem. Soc., 2003, 125, 8694-8695.

13 N. Ishikawa, M. Sugita, T. Ishikawa, S. Koshihara and Y. Kaizu, J. Phys. Chem. B, 2004, 108, 11265-11271.

14 M. A. AlDamen, J. M. Clemente-Juan, E. Coronado, C. MartiGastaldo and A. Gaita-Arino, J. Am. Chem. Soc., 2008, 130, 8874-8875. 
15 M. A. AlDamen, S. Cardona-Serra, J. M. Clemente-Juan, E. Coronado, A. Gaita-Arino, C. Marti-Gastaldo, F. Luis and O. Montero, Inorg. Chem., 2009, 48, 3467-3479.

16 M. Gonidec, F. Luis, À. Vílchez, J. Esquena, D. B. Amabilino and J. Veciana, Angew. Chem., Int. Ed., 2010, 49, 1623-1626.

17 S. Osa, T. Kido, N. Matsumoto, N. Re, A. Pochaba and J. Mrozinski, J. Am. Chem. Soc., 2004, 126, 420-421.

18 C. M. Zaleski, E. C. Depperman, J. W. Kampf, M. L. Kirk and V. L. Pecoraro, Angew. Chem., Int. Ed., 2004, 43, 3912-3914.

19 A. Mishra, W. Wernsdorfer, K. A. Abboud and G. Christou, J. Am. Chem. Soc., 2004, 126, 15648-15649.

20 J. P. Costes, J. M. Clemente-Juan, F. Dahan and J. Milon, Inorg. Chem., 2004, 43, 8200-8202.

21 C. Aronica, G. Pilet, G. Chastanet, W. Wernsdorfer, J. F. Jacquot and D. Luneau, Angew. Chem., Int. Ed., 2006, 45, 4659-4662.

22 J. P. Costes, F. Dahan and W. Wernsdorfer, Inorg. Chem., 2006, 45, 5-7.

23 M. Ferbinteanu, T. Kajiwara, K.-Y. Choi, H. Nojiri, A. Nakamoto, N. Kojima, F. Cimpoesu, Y. Fujimura, S. Takaishi and M. Yamashita, J. Am. Chem. Soc., 2006, 128, 9008-9009.

24 M. Murugesu, A. Mishra, W. Wernsdorfer, K. A. Abboud and G. Christou, Polyhedron, 2006, 25, 613-625.

25 F. Pointillart, K. Bernot, R. Sessoli and D. Gatteschi, Chem.-Eur. J., 2007, 13, 1602-1609.

26 M. Andruh, J. P. Costes, C. Diaz and S. Gao, Inorg. Chem., 2009, 48, 3342-3359.

27 J. Rinck, G. Novitchi, W. Van den Heuvel, L. Ungur, Y. H. Lan, W. Wernsdorfer, C. E. Anson, L. F. Chibotaru and A. K. Powell, Angew. Chem., Int. Ed., 2010, 49, 7583-7587.

28 T. Kajiwara, M. Nakano, K. Takahashi, S. Takaishi and M. Yamashita, Chem.-Eur. J., 2011, 17, 196-205.

29 J.-P. Sutter, S. B. Dhers, R. Rajamani, S. Ramasesha, J.-P. Costes, C. Duhayon and L. Vendier, Inorg. Chem., 2009, 48, 5820-5828.

30 J. Tang, I. Hewitt, N. T. Madhu, G. Chastanet, W. Wernsdorfer, C. E. Anson, C. Benelli, R. Sessoli and A. K. Powell, Angew. Chem., Int. Ed., 2006, 45, 1729-1733.
31 P.-H. Lin, T. Burchell, R. Clérac and M. Murugesu, Angew. Chem., Int. Ed., 2008, 47, 8848-8851.

32 L. F. Chibotaru, L. Ungur and A. Soncini, Angew. Chem., Int. Ed., 2008, 47, 4126-4129.

33 J. Luzon, K. Bernot, I. J. Hewitt, C. E. Anson, A. K. Powell and R. Sessoli, Phys. Rev. Lett., 2008, 100, 247205.

34 P.-H. Lin, T. J. Burchell, L. Ungur, L. F. Chibotaru, W. Wernsdorfer and M. Murugesu, Angew. Chem., Int. Ed., 2009, 48, 9489-9492.

35 I. Hewitt, J. Tang, N. Madhu, C. Anson, Y. Lan, J. Luzon, M. Etienne, R. Sessoli and A. Powell, Angew. Chem., Int. Ed., 2010, 49, 6352-6356.

36 R. A. Layfield, J. J. W. McDouall, S. A. Sulway, F. Tuna, D. Collison and R. E. P. Winpenny, Chem.-Eur. J., 2010, 16, 4442-4446.

37 F.-S. Guo, J.-L. Liu, J.-D. Leng, Z.-S. Meng, Z.-J. Lin, M.-L. Tong, S. Gao, L. Ungur and L. F. Chibotaru, Chem.-Eur. J., 2011, 17, 2458-2466.

38 L. F. Chibotaru, L. Ungur, C. Aronica, H. Elmoll, G. Pilet and D. Luneau, J. Am. Chem. Soc., 2008, 130, 12445-12455.

39 L. Ungur, W. Van den Heuvel and L. F. Chibotaru, New J. Chem., 2009, 33, 1224-1230.

40 F. Aquilante, L. De Vico, N. Ferre, G. Ghigo, P. A. Malmqvist, P. Neogrady, T. B. Pedersen, M. Pitonak, M. Reiher, B. O. Roos, L. Serrano-Andres, M. Urban, V. Veryazov and R. Lindh, J. Comput. Chem., 2010, 31, 224-247.

41 B. Clerjaud, B. Lambert, T. Buch and R. Romestai, Phys. Lett. A, 1973, 42, 341-342.

42 A. D. Trifunac and J. P. Smith, Chem. Phys. Lett., 1980, 73, 94-97.

43 C. T. Rodgers, K. B. Henbest, P. Kukura, C. R. Timmel and P. J. Hore, J. Phys. Chem. A, 2005, 109, 5035-5041.

44 A. Abragam and B. Bleaney, Electron Paramagnetic Resonance of Transition Ions, Oxford University Press, Oxford, UK, 1970.

45 F. A. Cotton, Chemical Applications of Group Theory, John Wiley \& Sons, New York, 1990.

46 G. Herzberg, Molecular Spectra and Molecular Structure, D. Van Nostrand Comp., Princeton, 1966. 